\title{
BPS invariants for resolutions of polyhedral singularities
}

\author{
Jim Bryan and Amin Gholampour
}

June 21, 2018

\begin{abstract}
We study the BPS invariants of the preferred Calabi-Yau resolution of ADE polyhedral singularities $\mathbb{C}^{3} / G$ given by Nakamura's $G$-Hilbert schemes. Genus 0 BPS invariants are defined by means of the moduli space of torsion sheaves as proposed by Sheldon Katz [8]. We show that these invariants are equal to half the number of certain positive roots of an ADE root system associated to $G$. This is in agreement with the prediction given in [4] via Gromov-Witten theory.
\end{abstract}

\section{Introduction}

The BPS invariants of a Calabi-Yau threefold $Y$ were first defined by Gopakumar and Vafa in the context of $M$-theory [6]. These invariants are conjecturally closely related to the Gromov-Witten invariants of $Y$, and since their appearance, there has been much effort to put them into a rigorous mathematical framework. The most satisfying approaches so far have been proposed by Sheldon Katz [8] via studying the moduli space of torsion sheaves on $Y$, and by Rahul Pandharipande and Richard Thomas [9] by means of moduli space of stable pairs on $Y$. The first considers all curve classes but is restricted to genus zero invariants, while the second can be defined for all genera but is confined to irreducible curve classes. In this paper we take the first point of view.

For a finite subgroup $G$ of $S O(3)$, let

$$
Y=G-\operatorname{Hilb}\left(\mathbb{C}^{3}\right)
$$


be Nakamura's Hilbert scheme of $G$-clusters in $\mathbb{C}^{3}$. By [5]

$$
\pi: Y \rightarrow \mathbb{C}^{3} / G
$$

is a Calabi-Yau semi-small resolution of singularities of $\mathbb{C}^{3} / G$, where $\pi$ is the Hilbert-Chow morphism. In particular, the fiber of $\pi$ over the origin is 1-dimensional. Moreover, it is proven in [5] that the reduced fiber over the origin, denoted by $F$, is a connected configuration of rational curves, and there exists a natural bijection between irreducible components of $F$ and non-trivial irreducible representations of $G$. It is shown in [4], that $Y$ can be realized as a surface fibration over $\mathbb{C}$. The central fiber, $S_{0}$, is a surface with finite number of ordinary double point singularities and $F \subset S_{0}$.

Let $\widehat{G}$ be the pullback of $G$ under the natural double cover map $S U(2) \rightarrow$ $S O(3)$, and let

$$
S=\widehat{G}-\operatorname{Hilb}\left(\mathbb{C}^{2}\right) .
$$

$S$ is a smooth surface and is the resolution of the ADE type singularity $\mathbb{C}^{2} / \widehat{G}$. McKay correspondence gives a natural bijection between irreducible components of the exceptional curve on $S$, which we denote by $\widehat{F}$, and nontrivial irreducible representations of $\widehat{G}$. By [3] there exists a regular projective morphism $f: S \rightarrow Y$ that factors through $S_{0}$ and it is the minimal resolution of singularities of $S_{0}$. Moreover $f$ maps $\widehat{F}$ onto $F$ in such a way that it maps isomorphically a component of $\widehat{F}$ corresponding to a representation of $G$ and contracts a component of $\widehat{F}$ otherwise. In Figure 1 we show the ADE Dynkin diagrams associated to $\widehat{F}$ in each case. Black vertices stand for the curves that are contracted by $f$.

In order to define BPS invariants in this paper we make essential use of the natural $\mathbb{C}^{*}$-action on $Y$ that is induced from the its diagonal action on $\mathbb{C}^{3}$. This induces an action on $S$ with respect to which $f$ is equivariant. Note that $F, \widehat{F}$ and $S_{0}$ are invariant under these actions.

Let $L$ be a line bundle on $Y$ whose restriction to each irreducible component of $F$ has a positive degree. Let $\beta \in H_{2}(Y, \mathbb{Z})$ be a curve class, and let $M^{L}(Y, \beta)$ be the Simpson moduli space of $L$-stable coherent sheaves $\mathcal{F}$ of pure dimension 1 on $Y$, with $\chi(\mathcal{F})=1$ and $\operatorname{ch}_{2}(\mathcal{F})=\beta$. Katz uses the moduli space $M^{L}(Y, \beta)$ to define the genus 0 BPS invariants of $Y$ in class $\beta$. Since $Y$ is a Calabi-Yau threefold the Hilbert polynomial of any $\mathcal{F}$ of pure dimension 1 is

$$
P_{\mathcal{F}}(n)=\left(L \cdot \operatorname{ch}_{2}(\mathcal{F})\right) n+\chi(\mathcal{F}) .
$$

By the condition $\chi(\mathcal{F})=1$, semi-stability implies stability, hence $M^{L}(Y, \beta)$ admits a perfect obstruction theory by [10] and hence a zero dimensional virtual cycle. $M^{L}(Y, \beta)$ is not necessarily compact because $Y$ is not, however 


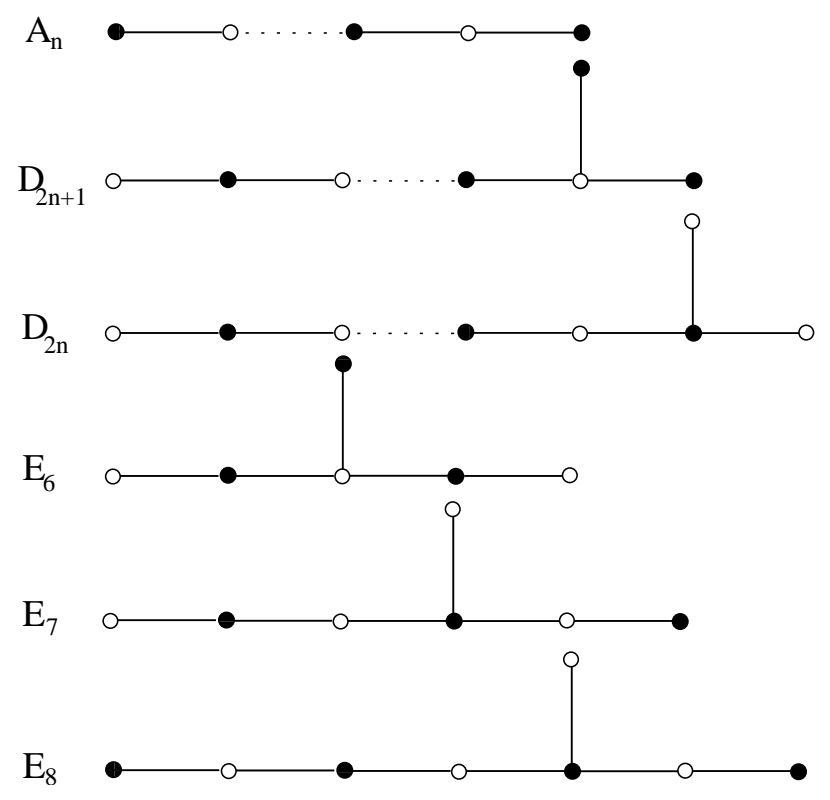

Figure 1: ADE Dynkin diagrams

it carries a $\mathbb{C}^{*}$-action that is induced from the action on $Y$; the fixed locus of this action is compact (see Theorem 1.1), so we can define BPS invariants as equivariant residues of this virtual cycle at the fixed locus.

Irreducible components of $F$ (respectively, of $\widehat{F}$ ) represent a basis for $H_{2}(Y, \mathbb{Z})$ (respectively, for $H_{2}(S, \mathbb{Z})$ ). The McKay correspondence gives a natural identification of $H_{2}(S, \mathbb{Z})$ with the associated ADE root lattice denoted by $R$. This induces a map $c: R^{+} \rightarrow H_{2}(Y, \mathbb{Z})\left(\right.$ via $f_{*}$ ) where $R^{+} \subset R$ is the set of positive roots (see [4] for more details). We say that $\beta \in H_{2}(Y, \mathbb{Z})$ corresponds to a positive root if it is in the image of $c$.

One of the main result of this paper is determining the fixed locus of the moduli space of BPS invariants:

Theorem 1.1. The $\mathbb{C}^{*}$-fixed point set of $M^{L}(Y, \beta)$ consists of a single point if $\beta$ corresponds to a positive root and is empty otherwise. Moreover, the sheaf corresponding to the fixed point in $M^{L}(Y, \beta)$ is $\mathcal{O}_{C}$, the structure sheaf of a certain Cohen-Macaulay curve $C \subset Y$, which we define in $\S 2$

This theorem is proven in $\S 2$. Computing equivariant residues of the virtual class at the fixed point given by Theorem 1.1, we can show: 
Theorem 1.2. Let $\beta \in H_{2}(Y, Z)$, and let $n_{\beta}^{L}(Y)$ denote the genus 0 BPS invariants of $Y$ in class $\beta$ (see $\S$ 3). Then

$$
n_{\beta}^{L}(Y)= \begin{cases}\frac{1}{2}\left|c^{-1}(\beta)\right| & \beta \text { corresponds to a positive root } \\ 0 & \text { otherwise }\end{cases}
$$

This result is in agreement with the prediction of $n_{\beta}^{L}(Y)$ in [4] obtained by means of Gromov-Witten theory.

Corollary 1.3. The genus 0 Gopakumar-Vafa conjecture [8] Conj 2.3] holds for $Y$.

Note that Theorem 1.2 shows that $n_{\beta}^{L}(Y)$ does not depend on the choice of polarization $L$. Proof of Theorem 1.2 is given in $\S 3$, in the most elaborate case, i.e. $Y=A_{5}-\mathrm{Hilb}\left(\mathbb{C}^{3}\right)$ and $\beta$ corresponds to the longest root in $E_{8}$ root system.

\section{Proof of Theorem 1.1}

Let $F_{1}, \ldots, F_{r}$ be the irreducible components of $F$. We know each $F_{i} \cong \mathbb{P}^{1}$; let $\alpha_{i}=\operatorname{deg}\left(\left.L\right|_{F_{i}}\right)$, note that $\alpha_{i}>0$ by the choice of $L$.

Lemma 2.1. Suppose $\mathcal{F} \in M^{L}(Y, \beta)$ is $\mathbb{C}^{*}$-fixed, then $\operatorname{supp}(\mathcal{F})$ is contained in $S_{0}$.

Proof. Since $F_{1}, \ldots, F_{r}$ are the only 1 dimensional invariant subvarieties of $Y$

$$
\operatorname{supp}(\mathcal{F})_{\text {reduced }} \subseteq F \subset S_{0} .
$$

$S_{0} \subset Y$ is a Cartier divisor with trivial normal bundle, hence there is an equivariant short exact sequence

$$
0 \rightarrow \mathcal{O}_{Y} \rightarrow \mathcal{O}_{Y} \rightarrow \mathcal{O}_{S_{0}} \rightarrow 0
$$

Tensoring with $\mathcal{F}$, we get

$$
\mathcal{F} \rightarrow \mathcal{F} \rightarrow \mathcal{F} \otimes \mathcal{O}_{S_{0}} \rightarrow 0
$$

Since $\mathcal{F}$ is stable the first map is either zero or an isomorphism. In the later case we conclude $\mathcal{F} \otimes \mathcal{O}_{S_{0}}=0$ which is impossible by (1). Hence the first map in the exact sequence above must be zero which implies $\mathcal{F} \cong \mathcal{F} \otimes \mathcal{O}_{S_{0}}$, and this proves the lemma. 
Since $\mathcal{F}$ is pure, $\operatorname{supp}(\mathcal{F})$ is a Cohen-Macaulay curve. Note that $\operatorname{supp}(\mathcal{F})$ is uniquely determined by these further conditions (see Lemma 3.2.i. in [8] for a similar situation):

(i) $\operatorname{supp}(\mathcal{F})$ is a subscheme of $S_{0}$,

(ii) $\operatorname{ch}_{2}(\mathcal{F})=\beta$.

The second condition determines the multiplicity of $\operatorname{supp}(\mathcal{F})$ at a generic point of each $F_{i}$ (away from the singularities of $S_{0}$ ). This number is welldefined and from now on we refer to it simply as multiplicity along $F_{i}$.

Lemma 2.2. Suppose $\mathcal{F} \in M^{L}(Y, \beta)$ is $\mathbb{C}^{*}$-fixed, then $\mathcal{F} \cong \mathcal{O}_{C}$ where $C$ is a Cohen-Macaulay curve.

Proof. Let $C=\operatorname{supp}(\mathcal{F})$. We know $C_{\text {reduced }} \subseteq F$ because $\mathcal{F}$ is $\mathbb{C}^{*}$-fixed. Since $\mathcal{F}$ is 1 dimensional, $\chi(\mathcal{F})=1$ implies that $\mathcal{F}$ has a global section $s: \mathcal{O}_{Y} \rightarrow \mathcal{F} . \operatorname{ker}(s)$ is the ideal sheaf of a subscheme $Z$ of $C$. We have $\mathcal{O}_{Z}=\operatorname{im}(s)$ is a subsheaf of $\mathcal{F}$, hence by purity of $\mathcal{F}, Z$ has to be a CohenMacaulay curve whose reduced support is a subset of $F$. We will show that $\mathcal{O}_{Z}$ will be a destabilizing subsheaf unless $Z=C$ and $\mathcal{F} \cong \mathcal{O}_{C}$.

To see this, let $m_{i}$ (respectively $m_{i}^{\prime}$ ) be the multiplicity of $C$ (respectively $Z$ ) along $F_{i}$. Since $\mathcal{O}_{Z}$ is a subsheaf of $\mathcal{F}$ we have $m_{i}^{\prime} \leq m_{i}$. By lemma 2.4 proven below, we know $\chi\left(\mathcal{O}_{Z}\right) \geq 1$, and by stability of $\mathcal{F}$ we must have

$$
\frac{\chi\left(\mathcal{O}_{Z}\right)}{\sum m_{i}^{\prime} \alpha_{i}} \leq \frac{1}{\sum m_{i} \alpha_{i}}
$$

which is impossible unless $Z=C$ and $\mathcal{F} \cong \mathcal{O}_{C}$.

Lemma 2.3. Suppose $C \subset Y$ is a proper, Cohen-Macaulay curve supported on $S_{0}$ with $\chi\left(\mathcal{O}_{C}\right)=1$, then $\mathcal{O}_{C}$ is $\mathbb{C}^{*}$-fixed and stable.

Proof. Since $C$ is proper $C_{\text {reduced }} \subseteq F$ and hence $\mathcal{O}_{C}$ is $\mathbb{C}^{*}$-fixed. To prove the lemma we check the stability condition for $\mathcal{O}_{C}$ (See [7, Proposition 1.2.6.iv]). Suppose $\mathcal{I}$ is a subsheaf of $\mathcal{O}_{C}$. $\mathcal{I}$ determines a subscheme $Z$ of $C$. If $Z$ is zero dimensional there is nothing to prove, so assume that $Z$ is one dimensional. Let $m_{i}$ be the multiplicity of $C$ along $F_{i}$. Let $C^{\prime}$ be a proper Weil divisor on $S_{0}$ having multiplicity $m_{i}^{\prime}$ along $F_{i}$, where $m_{i}^{\prime}$ is the generic multiplicity of $Z$ along $F_{i}$. Since $Z$ is a subscheme of $C$ we have $m_{i}^{\prime} \leq m_{i}$. By Lemma 2.4 below, $\chi\left(\mathcal{O}_{C^{\prime}}\right) \geq 1$, and hence $\chi\left(\mathcal{O}_{Z}\right) \geq 1$, because $Z$ differs from $C^{\prime}$ at possibly a finite number of points which they add up to its Euler characteristic. Thus

$$
\frac{\chi\left(\mathcal{O}_{Z}\right)}{\sum m_{i}^{\prime} \alpha_{i}} \geq \frac{1}{\sum m_{i} \alpha_{i}}
$$


with the equality only if $Z=C$. This proves the stability of $\mathcal{O}_{C}$, and the claim follows.

Theorem 1.1 follows from Lemmas 2.1, 2.2 and 2.3 so to complete the proof it remains to prove the follow lemma which was quoted above.

Lemma 2.4. Let $Z$ be a proper effective Weil divisor on $S_{0}$. Then $\chi\left(\mathcal{O}_{Z}\right) \geq$ 1 and equality holds if and only if the homology class of $Z$ corresponds to a positive root.

Proof. An effective Weil divisor $\widehat{Z}$ on $S$ which is supported on $\widehat{F}$, determines an element of $R^{+}$via McKay correspondence. By the adjunction formula, we have

$$
\chi\left(\mathcal{O}_{\widehat{Z}}\right)=-\widehat{Z}^{2} / 2 .
$$

Since $S$ is the resolution of rational singularities by [1, Proposition 1]

$$
\chi\left(\mathcal{O}_{\widehat{Z}}\right) \geq 1
$$

and the equality holds if and only if $\widehat{Z}$ corresponds to a positive root.

The idea of our proof is to relate $\chi\left(\mathcal{O}_{Z}\right)$ to $\chi\left(\mathcal{O}_{\widehat{Z}}\right)$ where $\widehat{Z} \subset S$ is defined as follows. Suppose

$$
Z=\sum_{i=1}^{r} m_{i} F_{i}
$$

we define a divisor

$$
\widehat{Z}=\sum_{i=1}^{r} m_{i} \widehat{F}_{i}+\sum_{j=1}^{s} n_{j} E_{j}
$$

on $S$ where $\widehat{F}_{i}$ is the proper transform of $F_{i}$ via $f: S \rightarrow S_{0}$,

$$
E_{1}, \ldots, E_{s}
$$

are exceptional curves of $f$, and $n_{j}$ is given as follows. Let

$$
k_{j}=E_{j} \cdot \sum_{i} m_{i} \widehat{F}_{i}
$$

be the sum of the multiplicities of the curves incident to $E_{j}$. We define

$$
n_{j}=\left\lceil\frac{k_{j}}{2}\right\rceil .
$$


Note that

$$
\delta_{j}=\widehat{Z} \cdot E_{j}=-2 n_{j}+k_{j}
$$

is either 0 or -1 by construction.

Now we claim that $\chi\left(\mathcal{O}_{Z}\right)=\chi\left(\mathcal{O}_{\widehat{Z}}\right)$. We show that $\operatorname{im}(\widehat{Z})$, the scheme theoretic image of $\widehat{Z}$ under $f$, is equal to $Z$. Since $f$ is an isomorphism away from singularities it suffices to show that $\operatorname{im}(\widehat{Z})$ does not carry any embedded points at singularities. Let $Z^{\prime}$ be a minimal divisor supported on $F$ such that $C=Z+Z^{\prime}$ is a Cartier divisor. By the choice of $\widehat{Z}$ one can see that $f^{*} C=\widehat{Z}+\widehat{Z}^{\prime}$, where $\widehat{Z}^{\prime}$ is the proper transform of $Z^{\prime}$. In fact they are linearly equivalent divisors on $S$ and are isomorphic away from exceptional curves. Since $f^{*} C$ is locally cut out by a single equation it is obvious that $C=\operatorname{im}\left(f^{*} C\right)$. But

$$
\operatorname{im}(\widehat{Z}) \subseteq \operatorname{im}\left(f^{*} C\right),
$$

and $C$, being locally cut out by a single equation on $S_{0}$ does not have any embedded points. This shows that $\operatorname{im}(\widehat{Z})$ does not have any embedded points either and hence $\operatorname{im}(\widehat{Z})=Z$. Since $S$ is a resolution of rational singularities $R^{1} f_{*} \mathcal{O}_{S}=0$, and moreover $R^{2} f_{*} \mathcal{F}=0$ for any coherent sheaf $\mathcal{F}$ on $S$, so we get immediately $R^{1} f_{*} \mathcal{O}_{\widehat{Z}}=0$, and therefore

$$
\chi\left(\mathcal{O}_{\widehat{Z}}\right)=\chi\left(f_{*} \mathcal{O}_{\widehat{Z}}\right)
$$

by Leray spectral sequence. Since $f_{*} \mathcal{O}_{S} \cong \mathcal{O}_{S_{0}}$ one can see the isomorphism of ideal sheaves

$$
\mathcal{I}_{Z}=\mathcal{I}_{\operatorname{im}(\widehat{Z})} \cong f_{*} \mathcal{I}_{\widehat{Z}}
$$

and hence we have a short exact sequence

$$
0 \rightarrow \mathcal{I}_{Z} \rightarrow \mathcal{O}_{S_{0}} \rightarrow f_{*} \mathcal{O}_{\widehat{Z}} \rightarrow 0 .
$$

Here we used the fact that $R^{1} f_{*}\left(\mathcal{I}_{\widehat{Z}}\right)=0$ which is true because the restriction of $\mathcal{I}_{\widehat{Z}} \cong \mathcal{O}_{S}(-\widehat{Z})$ on each $E_{j}$ is equal to $-\delta_{j}$ which is non-negative. The short exact sequence above implies that $f_{*} \mathcal{O}_{\widehat{Z}} \cong \mathcal{O}_{Z}$. Now the claim follows from (3).

To finish the proof of lemma we only need to show if the homology class of $Z$ corresponds to a positive root then $\widehat{Z}$ is a positive root. Since $n_{j} \geq 0$, $\widehat{Z}$ is a positive root if and only if $\widehat{Z}^{2}=-2$.

Let

$$
\widetilde{Z}=\sum_{i} m_{i} \widehat{F}_{i}+\sum_{j} \widetilde{n}_{j} E_{j}
$$


be any positive root which corresponds to $Z$. Since $\widetilde{Z}$ and $E_{j}$ are distinct positive roots in an ADE root system,

$$
\widetilde{\delta}_{j}=\widetilde{Z} \cdot E_{j}
$$

is equal to $-1,0$, or 1 . We compute

$$
\begin{aligned}
\widehat{Z}^{2}-\widetilde{Z}^{2} & =(\widehat{Z}-\widetilde{Z}) \cdot(\widehat{Z}+\widetilde{Z}) \\
& =\sum_{j}\left(n_{j}-\widetilde{n}_{j}\right)\left(\delta_{j}+\widetilde{\delta}_{j}\right) .
\end{aligned}
$$

Since

$$
\begin{aligned}
& \delta_{j}=E_{j} \cdot \widehat{Z}=-2 n_{j}+k_{j}, \\
& \widetilde{\delta}_{j}=E_{j} \cdot \widetilde{Z}=-2 \widetilde{n}_{j}+k_{j}
\end{aligned}
$$

we get

$$
n_{j}-\widetilde{n}_{j}=\frac{1}{2}\left(\widetilde{\delta}_{j}-\delta_{j}\right)
$$

and therefore

$$
\widehat{Z}^{2}-\widetilde{Z}^{2}=\sum_{j} \frac{1}{2}\left(\widetilde{\delta}_{j}^{2}-\delta_{j}^{2}\right)
$$

Since $n_{j}-\widetilde{n}_{j}$ is an integer, we know that $\delta_{j}$ is congruent to $\widetilde{\delta}_{j}$ modulo 2 . Then since $\delta_{j}, \widetilde{\delta}_{j} \in\{-1,0,1\}$, we conclude that

$$
\widetilde{\delta}_{j}^{2}-\delta_{j}^{2}=0
$$

so $\widehat{Z}^{2}=\widetilde{Z}^{2}=-2$, and hence $\widehat{Z}$ is a positive root.

\section{BPS invariants}

As mentioned in $\S$ 1. BPS invariants of $Y$ are defined as the equivariant residues at the $\mathbb{C}^{*}$-fixed locus of the corresponding moduli space. More precisely, let $\beta \in H_{2}(Y, \mathbb{Z})$. By Theorem 1.1 we already know that the fixed locus is either empty or a single point, and the later happens only when $\beta$ corresponds to a positive root. In fact if

$$
\beta=\sum_{i=1}^{r} m_{i}\left[F_{i}\right]
$$


is such a curve class, then we proved this fixed point of moduli space is given by $\mathcal{O}_{C}$ the structure sheaf of a 1-dimensional Cohen-Macaulay scheme having generic multiplicity $m_{i}$ along $F_{i}$. We also showed $C$ is moreover scheme-theoretically supported on $S_{0}$. Now fix such a class $\beta$ and the curve $C$ determined as above. The corresponding BPS invariant is by definition

$$
n_{\beta}^{L}(Y)=\frac{e\left(\operatorname{Ext}_{Y}^{2}\left(\mathcal{O}_{C}, \mathcal{O}_{C}\right)\right)}{e\left(\operatorname{Ext}_{Y}^{1}\left(\mathcal{O}_{C}, \mathcal{O}_{C}\right)\right)}
$$

where $e(-)$ is the equivariant Euler class.

For any nonzero integer $a$ by $\mathbb{C}_{a}$ we mean the weight $a$ representation of $\mathbb{C}^{*}$. The canonical bundle of $Y$, is trivial with weight -3 . We have

$$
\operatorname{Hom}_{\mathrm{Y}}\left(\mathcal{O}_{C}, \mathcal{O}_{C}\right) \cong \mathbb{C} \text { and } \operatorname{Ext}_{Y}^{3}\left(\mathcal{O}_{C}, \mathcal{O}_{C}\right) \cong \mathbb{C}_{3},
$$

where the first isomorphism is because of the stability of $\mathcal{O}_{C}$ and the second is because of equivariant Serre duality. Let

$$
\chi\left(\mathcal{O}_{C}, \mathcal{O}_{C}\right)=\sum_{p=0}^{3}(-1)^{p} \operatorname{Ext}_{Y}^{p}\left(\mathcal{O}_{C}, \mathcal{O}_{C}\right)
$$

be the Euler characteristic where the right hand side is regarded as a virtual $\mathbb{C}^{*}$ representation. Since we already know the first and the last term contributions, if we find $\chi\left(\mathcal{O}_{C}, \mathcal{O}_{C}\right)$ we will be able to determine $n_{\beta}^{L}(Y)$ (note we only need the ratio of the Euler classes of the third and second terms).

By using equivariant Hirzebruch-Riemann-Roch we have (see [7, Lemma 6.1.3])

$$
\operatorname{ch}\left(\chi\left(\mathcal{O}_{C}, \mathcal{O}_{C}\right)\right)=\int_{Y} \operatorname{ch}^{\vee}\left(\mathcal{O}_{C}\right) \operatorname{ch}\left(\mathcal{O}_{C}\right) \operatorname{td}(Y)
$$

where

$$
\operatorname{ch}=\operatorname{ch}_{0}+\operatorname{ch}_{1}+\operatorname{ch}_{2}+\cdots
$$

is the equivariant Chern character and

$$
\operatorname{ch}^{\vee}=\operatorname{ch}_{0}-\operatorname{ch}_{1}+\operatorname{ch}_{2}-\cdots .
$$

$\operatorname{td}(-)$ denotes the equivariant Todd class. By this integral we mean equivariant push-forward from $Y$ to a point.

One way to compute $\operatorname{ch}\left(\mathcal{O}_{C}\right)$ is to find an equivariant locally free resolution of $\mathcal{O}_{C}$. However, in most of our cases $C$ is not a local complete intersection in $Y$ and so it is difficult to find such a resolution for $\mathcal{O}_{C}$ in such cases. We use an alternative method to compute equivariant Chern 
characters of $\mathcal{O}_{C}$ at the components of fixed locus of $Y$. By Atiyah-Bott residue formula, this is all we need to know in order to evaluate the integral in (4). As in the proof of Lemma 2.4 we can construct a divisor $\widehat{C}$ on $S$ invariant under the induced $\mathbb{C}^{*}$-action and with the property $f_{*} \mathcal{O}_{\widehat{C}}=\mathcal{O}_{C}$ and $R^{i} f_{*} \mathcal{O}_{\widehat{C}}=0$ for $i>0$. Applying Grothendieck-Riemann-Roch to the equivariant projective morphism $f: S \rightarrow Y$ we have this relation in the equivariant $K$-group of $Y$ :

$$
\operatorname{ch}\left(\mathcal{O}_{C}\right)=\frac{1}{\operatorname{td}(Y)} f_{*}\left(\operatorname{ch}\left(\mathcal{O}_{\widehat{C}}\right) \cdot \operatorname{td}(S)\right) .
$$

Since $\widehat{C}$ is a divisor on a smooth surface, there is a natural locally free resolution for $\mathcal{O}_{\widehat{C}}$ :

$$
0 \rightarrow \mathcal{O}_{S}(-\widehat{C}) \rightarrow \mathcal{O}_{S} \rightarrow \mathcal{O}_{\widehat{C}} \rightarrow 0 .
$$

Let $i_{P}: P \hookrightarrow Y$ be a $\mathbb{C}^{*}$-fixed component. By correspondence of residues (e.g. see $[2, \S 3]$ ) we can write

$$
i_{P}^{*}\left(\operatorname{ch}\left(\mathcal{O}_{C}\right)\right)=\frac{e\left(N_{P \mid Y}\right)}{i_{P}^{*} \operatorname{td}(Y)} \cdot \sum_{Q \rightarrow P} j_{Q}^{*} f_{*}\left(\left(1-\operatorname{ch}\left(\mathcal{O}_{S}(-\widehat{C})\right) \cdot \frac{\operatorname{td}(S)}{e\left(N_{Q \mid S}\right)}\right)\right.
$$

where $j_{Q}: Q \hookrightarrow S$ is a $\mathbb{C}^{*}$-fixed component in $S$, and the sum is over all such components mapping into $P$.

Our proof of Theorem 1.2 is a case by case check using (4) and (5). We carry this out for some of the most complicated roots in the case corresponding to $E_{8}$; all other cases are similar and simpler. The $E_{8}$ root system has 120 positive roots and this leads to 36 curve classes on $Y$ having nonzero BPS invariants. The possible values of the nonzero invariants and the number of classes carrying these invariants and the number of positive roots corresponding to these classes are summarized below:

\begin{tabular}{|c|c|c|}
\hline$\sharp$ of positive roots & $\sharp$ of classes $\beta$ & $n_{\beta}(Y)$ \\
\hline 32 & 16 & 1 \\
\hline 4 & 4 & $\frac{1}{2}$ \\
\hline 48 & 12 & 2 \\
\hline 32 & 4 & 4 \\
\hline
\end{tabular}

Note that the entries of the first column add up to 116, because there are 4 positive simple roots that do not correspond to a curve class on $Y$. These simple roots correspond to black vertices of the $E_{8}$ Dynkin diagram in Figure 1

In the following proposition we list the largest class $\beta$ carrying each possible nonzero invariant. 


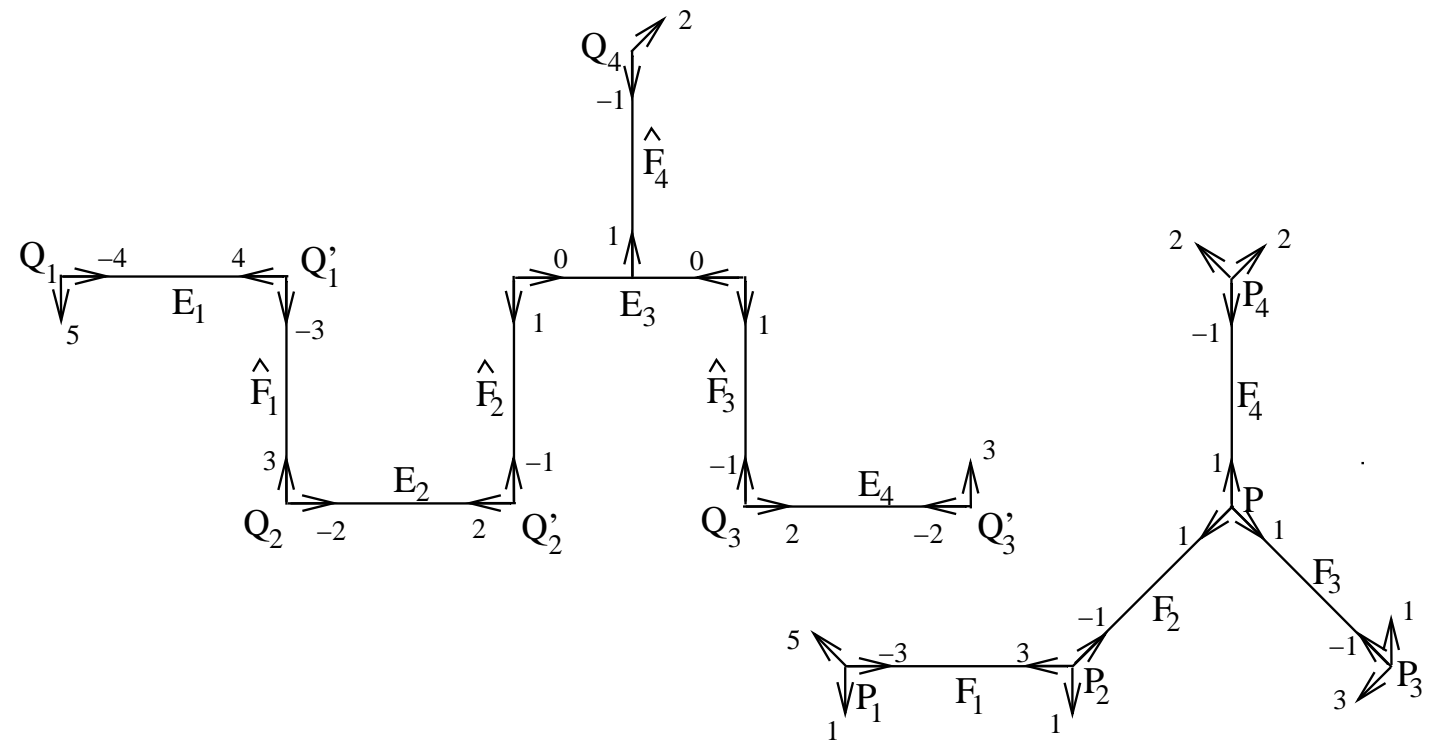

Figure 2: The fibers over origin on $\widehat{A}_{5}-\mathrm{Hilb}\left(\mathbb{C}^{2}\right)$ (left) and in $A_{5}-\mathrm{Hilb}\left(\mathbb{C}^{3}\right)$ (right)

Proposition 3.1. Let $G=A_{5}$ be the alternating group in 5 elements; this case corresponds to the root system $E_{8}$. Let $F_{1}, \ldots, F_{4}$ be the components of $F$ as in Figure 2. Then

$\operatorname{ch}\left(\chi\left(\mathcal{O}_{C}, \mathcal{O}_{C}\right)\right)= \begin{cases}1-\mathrm{e}^{3 t} & C=3 F_{1}+5 F_{2}+4 F_{3}+3 F_{4} \\ 1-\mathrm{e}^{2 t}+\mathrm{e}^{t}-\mathrm{e}^{3 t} & C=2 F_{1}+4 F_{2}+4 F_{3}+2 F_{4} \\ 1-\mathrm{e}^{t}+\mathrm{e}^{2 t}-\mathrm{e}^{3 t} & C=2 F_{1}+4 F_{2}+3 F_{3}+2 F_{4} \\ 1-2 \mathrm{e}^{t}+2 \mathrm{e}^{2 t}-\mathrm{e}^{3 t} & C=F_{1}+2 F_{2}+2 F_{3}+F_{4}\end{cases}$

where $t$ is the equivariant parameter.

The following corollary is now immediate:

Corollary 3.2. Let $G$ and $F_{1}, \ldots, F_{4}$ be as in proposition and let $\beta=$ $k_{1}\left[F_{1}\right]+k_{2}\left[F_{2}\right]+k_{3}\left[F_{3}\right]+k_{4}\left[F_{4}\right]$. Then

\begin{tabular}{|c|c|c|c|}
\hline$\left(k_{1}, k_{2}, k_{3}, k_{4}\right)$ & $e\left(\operatorname{Ext}_{Y}^{2}\left(\mathcal{O}_{C}, \mathcal{O}_{C}\right)\right)$ & $e\left(\operatorname{Ext}_{Y}^{1}\left(\mathcal{O}_{C}, \mathcal{O}_{C}\right)\right)$ & $n_{\beta}(Y)$ \\
\hline$(3,5,4,3)$ & 1 & 1 & 1 \\
\hline$(2,4,4,2)$ & $t$ & $2 t$ & $\frac{1}{2}$ \\
\hline$(2,4,3,2)$ & $2 t$ & $t$ & 2 \\
\hline$(1,2,2,1)$ & $(2 t)^{2}$ & $t^{2}$ & 4 \\
\hline
\end{tabular}


The values in the last column are as given in Theorem 1.2 Note that the values given in the second and the third columns are modulo possibly cancelling factors common in two Euler classes.

Proof of Proposition. In Figure 2, $P_{1}, P_{2}, P_{3}, P_{4}, P$ and

$$
Q_{1}, Q_{1}^{\prime}, Q_{2}, Q_{2}^{\prime}, Q_{3}, Q_{3}^{\prime}, Q_{4}, E_{3}
$$

are the $\mathbb{C}^{*}$-fixed components of

$$
Y=A_{5}-\operatorname{Hilb}\left(\mathbb{C}^{3}\right) \text { and } S=\widehat{A}_{5}-\operatorname{Hilb}\left(\mathbb{C}^{2}\right),
$$

respectively. $f$ maps $Q_{i}$ and $Q_{i}^{\prime}$ to $P_{i}$ and $E_{3}$ to $P$. The numbers in this figure stand for $\mathbb{C}^{*}$-weights of the tangent spaces at the fixed points. We only prove the first identity; the proof of the rest is similar. In this case

$$
\widehat{C}=3 \widehat{F}_{1}+5 \widehat{F}_{2}+4 \widehat{F}_{3}+3 \widehat{F}_{4}+2 E_{1}+4 E_{2}+6 E_{3}+2 E_{4} .
$$

Note that this corresponds to the longest root in the root system $E_{8}$. Using (4) and (5), we compute the contribution of each $\mathbb{C}^{*}$-fixed component of $Y$ to $\operatorname{ch}\left(\chi\left(\mathcal{O}_{C}, \mathcal{O}_{C}\right)\right)$. We denote by $\chi_{i}$ the contribution of $P_{i}$, and by $\chi$ the contribution of $P$. For simplicity, we let $\mu=\mathrm{e}^{t}$, and $\operatorname{ch}(P)=i_{P}^{*} \operatorname{ch}\left(\mathcal{O}_{C}\right)$ and $\operatorname{ch}(Q)=j_{Q}^{*} \operatorname{ch}\left(\mathcal{O}_{\widehat{C}}\right)$ and similarly for $P_{i}$ 's, $Q_{i}$ 's and $E_{3}$. We use the same notation for $\mathrm{ch}^{\vee}$. Finally by $\operatorname{ch}\left(Q_{i} \rightarrow P_{i}\right)$ we mean the contribution of $j_{Q_{i}}^{*} \operatorname{ch}\left(\mathcal{O}_{\widehat{C}}\right)$ to $i_{P_{i}}^{*} \operatorname{ch}\left(\mathcal{O}_{C}\right)$ in (5), and similarly for $\operatorname{ch}\left(Q_{i}^{\prime} \rightarrow P_{i}\right)$.

- Contribution of $P_{1}$ : We have $\operatorname{ch}\left(Q_{1}\right)=1-\mu^{-10}$,

$$
\begin{gathered}
\frac{i_{P_{1}}^{*} \operatorname{td}(Y)}{e\left(N_{P_{1} \mid Y}\right)}=\frac{1}{\left(1-\mu^{-5}\right)\left(1-\mu^{-1}\right)\left(1-\mu^{3}\right)} \quad \text { and } \\
\frac{j_{Q_{1}}^{*} \operatorname{td}(S)}{e\left(N_{Q_{1} \mid S}\right)}=\frac{1}{\left(1-\mu^{-5}\right)\left(1-\mu^{4}\right)} . \\
\text { Hence } \operatorname{ch}\left(Q_{1} \rightarrow P_{1}\right)=\frac{\left(1-\mu^{-10}\right)\left(1-\mu^{3}\right)\left(1-\mu^{-1}\right)}{\left(1-\mu^{4}\right)} \text { and similarly } \\
\operatorname{ch}\left(Q_{1}^{\prime} \rightarrow P_{1}\right)=\frac{\left(1-\mu^{-6}\right)\left(1-\mu^{-5}\right)\left(1-\mu^{-1}\right)}{\left(1-\mu^{-4}\right)} .
\end{gathered}
$$

In the last formula we used $\operatorname{ch}\left(Q_{1}^{\prime}\right)=1-\mu^{-6}$. So we have

$$
\begin{aligned}
\operatorname{ch}\left(P_{1}\right) & =\operatorname{ch}\left(Q_{1} \rightarrow P_{1}\right)+\operatorname{ch}\left(Q_{1}^{\prime} \rightarrow P_{1}\right) \\
& =\mu^{-11}-\mu^{-10}+\mu^{-7}-\mu^{-6}-\mu^{-2}+1
\end{aligned}
$$


and hence $\operatorname{ch}^{\vee}\left(P_{1}\right)=1-\mu^{2}-\mu^{6}+\mu^{7}-\mu^{10}+\mu^{11}$. We finally get the contribution of $P_{1}$ to (4):

$$
\begin{aligned}
\chi_{1} & =\frac{\operatorname{ch}^{\vee}\left(P_{1}\right) \cdot \operatorname{ch}\left(P_{1}\right) \cdot i_{P_{1}}^{*} t d(Y)}{e\left(N_{P_{1} \mid Y}\right)} \\
& =\mu^{-5}-\mu^{-3}+\mu^{-2}+\mu^{-1}-\mu+\mu^{2}-\mu^{4}-\mu^{5}+\mu^{6}-\mu^{8} .
\end{aligned}
$$

- Contributions of $P_{2}, P_{3}, P_{4}$ : These cases are quite similar to the case of $P_{1}$; we only summarize the results below:

$$
\begin{aligned}
& \operatorname{ch}\left(Q_{2} \rightarrow P_{2}\right)=\frac{\left(1-\mu^{-6}\right)\left(1-\mu^{-1}\right)(1-\mu)\left(1-\mu^{-3}\right)}{\left(1-\mu^{2}\right)\left(1-\mu^{-3}\right)}, \\
& \operatorname{ch}\left(Q_{2}^{\prime} \rightarrow P_{2}\right)=\frac{\left(1-\mu^{-6}\right)\left(1-\mu^{-1}\right)(1-\mu)\left(1-\mu^{-3}\right)}{\left(1-\mu^{-2}\right)(1-\mu)}, \\
& \operatorname{ch}\left(Q_{3} \rightarrow P_{3}\right)=\frac{\left(1-\mu^{-6}\right)\left(1-\mu^{-1}\right)(1-\mu)\left(1-\mu^{-3}\right)}{\left(1-\mu^{-2}\right)(1-\mu)}, \\
& \operatorname{ch}\left(Q_{3}^{\prime} \rightarrow P_{3}\right)=\frac{\left(1-\mu^{-6}\right)(1-\mu)\left(1-\mu^{-1}\right)\left(1-\mu^{-3}\right)}{\left(1-\mu^{2}\right)\left(1-\mu^{-3}\right)}, \\
& \operatorname{ch}\left(Q_{4} \rightarrow P_{4}\right)=\left(1-\mu^{-6}\right)\left(1-\mu^{-2}\right), \\
& \operatorname{ch}\left(P_{2}\right)=\mu^{-8}-\mu^{-6}-\mu^{-2}+1, \\
& \operatorname{ch}\left(P_{3}\right)=\mu^{-8}-\mu^{-6}-\mu^{-2}+1, \\
& \operatorname{ch}\left(P_{4}\right)=\mu^{-8}-\mu^{-6}-\mu^{-2}+1, \\
& \chi_{2}=\mu^{-4}+2 \mu^{-3}+\mu^{-2}+\mu^{-1}+2+\mu-\mu^{2}-2 \mu^{3}-\mu^{4}-\mu^{5}-2 \mu^{6}-\mu^{7}, \\
& \chi_{3}=\mu^{-4}+2 \mu^{-3}+\mu^{-2}+\mu^{-1}+2+\mu-\mu^{2}-2 \mu^{3}-\mu^{4}-\mu^{5}-2 \mu^{6}-\mu^{7}, \\
& \chi_{4}=\mu^{-4}+\mu^{-3}+\mu^{-2}+\mu^{-1}+1+\mu-\mu^{2}-\mu^{3}-\mu^{4}-\mu^{5}-\mu^{6}-\mu^{7} .
\end{aligned}
$$

- Contribution of $P$ : Note that $-\widehat{C} \cdot E_{3}=0$, and hence $\left.\mathcal{O}_{S}(-\widehat{C})\right|_{E_{3}}$ is trivial with weight -6 , $\operatorname{so} \operatorname{ch}\left(E_{3}\right)=1-\mu^{-6}$. One sees easily that

$$
\frac{i_{P}^{*} \operatorname{td}(Y)}{e\left(N_{P \mid Y}\right)}=\frac{1}{\left(1-\mu^{-1}\right)^{3}} \text {. }
$$

Let $[x]$ be the point class on $E_{3} \cong \mathbb{P}^{1}$ then

$$
\frac{j_{E_{3}}^{*} \operatorname{td}(S)}{e\left(N_{E_{3} \mid S}\right)}=\frac{2[x]}{\left(1-\mathrm{e}^{-t+2[x]}\right)\left(1-\mathrm{e}^{-2[x]}\right)}=\left(\frac{1}{1-\mu^{-1}}-\frac{2[x] \mu^{-1}}{\left(1-\mu^{-1}\right)^{2}}\right)(1+[x]) .
$$

So we get

$$
\begin{aligned}
\operatorname{ch}(P) & =\left(1-\mu^{-6}\right)\left(1-u^{-1}\right)^{3} \cdot f_{*}\left(\frac{(1+[x])}{1-\mu^{-1}}-\frac{2[x] \mu^{-1}}{\left(1-\mu^{-1}\right)^{2}}\right) \\
& =\mu^{-8}-\mu^{-6}-\mu^{-2}+1 .
\end{aligned}
$$


And finally we get

$$
\begin{aligned}
\chi & =\frac{\left(1-\mu^{2}-\mu^{6}+\mu^{8}\right)\left(\mu^{-8}-\mu^{-6}-\mu^{-2}+1\right)}{\left(1-\mu^{-1}\right)^{3}} \\
& =-\mu^{-5}-3 \mu^{-4}-4 \mu^{-3}-4 \mu^{-2}-4 \mu^{-1}-4-2 \mu+2 \mu^{2}+4 \mu^{3} \\
& +4 \mu^{4}+4 \mu^{5}+4 \mu^{6}+3 \mu^{7}+\mu^{8} .
\end{aligned}
$$

At the end we add all the contributions

$$
\operatorname{ch}\left(\chi\left(\mathcal{O}_{C}, \mathcal{O}_{C}\right)\right)=\chi+\chi_{1}+\chi_{2}+\chi_{3}+\chi_{4}=1-\mu^{3} .
$$

\section{References}

[1] Michael Artin. On isolated rational singularities of surfaces. Amer. J. Math., 88:129-136, 1966.

[2] Aaron Bertram. Another way to enumerate rational curves with torus actions. Invent. Math., 142(3):487-512, 2000. arXiv:math/9905159.

[3] Samuel Boissière and Alessandra Sarti. Contraction of excess fibres between the McKay correspondences in dimensions two and three. Ann. Inst. Fourier (Grenoble), 57(6):1839-1861, 2007. math.AG/math/0504360.

[4] Jim Bryan and Amin Gholampour. The Quantum McKay Correspondence for polyhedral singularities . arXiv:0803.3766.

[5] Yasushi Gomi, Iku Nakamura, and Ken-ichi Shinoda. Hilbert schemes of $G$-orbits in dimension three. Asian J. Math., 4(1):51-70, 2000. Kodaira's issue.

[6] Rajesh Gopakumar and Cumrun Vafa. M-theory and topological strings-II, 1998. Preprint, hep-th/9812127.

[7] Daniel Huybrechts and Manfred Lehn. The geometry of moduli spaces of sheaves. Friedr. Vieweg \& Sohn, Braunschweig, 1997.

[8] Sheldon Katz. Genus zero Gopakumar-Vafa invariants of contractible curves. J. Differential Geom., 79(2):185-195, 2008. arXiv:math/0601193.

[9] Richard Thomas Rahul Pandharipande. Stable pairs and BPS invariants . arXiv:0711.3899. 
[10] R. P. Thomas. A holomorphic Casson invariant for Calabi-Yau 3-folds, and bundles on K3 fibrations. J. Differential Geom., 54(2):367-438, 2000. arXiv:math/9806111.

Department of Mathematics, University of British Columbia

Department of Mathematics, California Institute of Technology

Email address: jbryan@math.ubc.ca, agholamp@ caltech.edu 